\title{
Suberoylanilide Hydroxamic Acid (SAHA) Treatment Reveals Crosstalk Among Proteome, Phosphorylome and Acetylome in Nasopharyngeal Carcinoma Cells
}

Huichao Huang ( $\square$ hhc19881121love@126.com )

Xiangya Hospital Central South University https://orcid.org/0000-0002-2522-847X

\section{Ying Fu}

Central South University

\section{Ye Zhang}

Central South University

\section{Fang Peng}

Central South University

Miaolong Lu

Central South University

\section{Zhuchu Chen}

Central South University

Maoyu Li

Central South University

\section{Yongheng Chen}

Central South University

\section{Research Article}

Keywords: Suberoylanilide Hydroxamic Acid (SAHA), Proteome, Acetylome, Phosphorylome, Nasopharyngeal carcinoma (NPC), Predictive preventive personalized medicine (PPPM)

Posted Date: July 20th, 2021

DOl: https://doi.org/10.21203/rs.3.rs-655483/v1

License: (9) (1) This work is licensed under a Creative Commons Attribution 4.0 International License. Read Full License 


\section{Abstract}

Suberoylanilide hydroxamic acid (SAHA), as a famous histone deacetylase (HDAC) inhibitor, has been utilized in clinical treatment for cutaneous T-cell lymphoma. Previously the mechanisms underlying SAHA anti-tumor mainly focused on acetylome. However, the characteristics of SAHA in terms of other protein posttranslational modifications (PTMs) and the crosstalk between various modifications are poorly understood. Our previous work revealed that SAHA has the anti-tumor activity in nasopharyngeal carcinoma (NPC) cells as well. In this study, we reported that the profiles of global proteome, acetylome and phosphorylome of 5-8F cells upon SAHA induction and the crosstalk between these data sets. Overall, we detected and quantified 6491 proteins, 2456 phosphorylated proteins and 228 acetylated proteins toward SAHA treatment in 5-8F cells. In addition, we identified 46 proteins which all exhibited both acetylation and phosphorylation. With the aid of intensive bioinformatics analyses, multiple cellular processes and signaling pathways involved in tumorigenesis and metabolism were clustered, including TCA cycle, glycolysis, EGFR signaling and Myc signaling pathways. Taken together, this study highlighted the interconnectivity of acetylation and phosphorylation signaling networks and suggested SAHA may influent cellular response by affecting both types of protein modifications in 5-8F cells. Further our work also paved the way for the predictive and personalized treatment of NPC patients.

\section{Introduction}

Histone deacetylases (HDACs) modulate gene expressions epigenetically via affecting protein acetylation status under diverse situations, thus play key roles in pathophysiological processes [1]. Since HDACs as well as protein acetylation contribute to tumorigenesis, specific inhibitors (HDACi) targeted HDACs have become a promising approach to treat tumor [2]. Suberoylanilide hydroxamic acid (SAHA, vorinostat)), a member of HDACi family, has been used clinically as first-line therapy for refractory cutaneous $T$ cell lymphomas (CTCL) $[3,4]$. In addition to CTCL, more and more researches have unveiled that SAHA also deploys anti-tumor activities in many other tumors, such as lung cancer, breast cancer, ovarian cancer, as well as head and neck tumors [5-9]. However, SAHA's anti-tumor property towards NPC cells is still elusive.

Protein posttranslational modifications (PTMs) are universal means to maintain fundamental biological functions in the body, including but not limited to gene expression, signal transduction and cell proliferation [10-12]. Phosphorylation, acetylation, methylation, glycosylation and ubiquitination these are common PTMs. The contribution of PTMs in tumorigenesis and progression has been reported [12, 13]. Phosphorylation refers to the attachment of phosphoryl group on the target amino acid and highly regulated, which is considered the most abundant PTM in eukaryotes. It is critical for many cellular processes in biology, along with dephosphorylation. For example, protein phosphorylation activates or deactivates almost half of the enzymes present in Saccharomyces cerevisiae, thereby regulating their function [14-16]. On the other hand, acetylation describes the process that transfers acetyl moiety from acetyl-CoA to its amino groups in lysine residues, which is enzymatically reversible and is tightly regulated by metabolism-dependent mechanisms. Acetylation and deacetylation interplay is the key to 
many important cellular processes, malfunctioning in this machinery can result in severe conditions such as cancer, neurodegenerative diseases and cardiovascular disorders [17]. It also has been revealed that acetylation and phosphorylation are closely linked and affect each other. Habibian $\mathrm{J}$ etc. reported that HDACi regulated crosstalk between acetylation and phosphorylation in the treatment of cardiac disease [18]. Previous works showed that protein tau underwent acetylation, phosphorylation and ubiquitination in the development of neurodegenerative diseases [19]. There are also studies revealed that both acetylation and phosphorylation occurred in p53 and regulated its activity and stability. For example, phosphorylation of p53 at Ser15 and Ser46 leads to the acetylation of p53 at Lys 382, which in turn induced cell apoptosis and cell cycle arrest [20].

In view of the extensive mediated roles of PTMs and the close relationship between acetylation and phosphorylation, it is reasonable to explore the potential property of SAHA's anti-tumor activity in NPC cells via PTMs and the crosstalk among diverse protein modifications. Besides, knowing the characteristics of SAHA in NPC cells is also consistent with the paradigm of PPPM, which is the integral strategy in healthcare [21, 22]. In the current work, we combined TMT labeling, antibody affinity enrichment and high-resolution LC-MS/ MS approaches to quantitatively compare the global proteome, phosphorylome and acetylome in 5-8F cell line with/without SAHA treatment. In addition, bioinformaticbased systematic analyses were applied to investigate the crosstalk among the three protein modifications. Collectively, we demonstrated that SAHA treatment dramatically regulated global proteome, phosphorylome and acetylome in 5-8F cell line. Meanwhile, our results showed that some key signaling pathways, cellular metabolic processes as well as widespread protein-protein interactions were modulated by phosphorylation and acetylation. Overall, this study provided a novel insight as to how SAHA exerted biological functions in NPC cells and presented the scientific data for effectively predictive and personalized treatment of NPC patients.

\section{Materials And Methods}

\section{Cell Culture}

Cells were all purchased from American Type Culture Collection and cultured as previously described. Briefly, 5-8F cells were cultured in RPMI- 1640 medium containing $10 \%$ fetal bovine serum (FBS) and $1 \%$ penicillin and streptomycin in a humidified environment at $37^{\circ} \mathrm{C}$ and $5 \% \mathrm{CO}_{2}$.

\section{Protein Extraction and Digestion}

Cells were harvested, then washed with ice cold PBS and lysed by incubation in SDS lysis buffer. After quantification, protein digestion was performed according to the filter-aided sample preparation (FASP) procedure. Briefly, 200 ug proteins were reduced with $100 \mathrm{mM}$ DTT and then 200ul UA buffer (8M Urea, $150 \mathrm{mM}$ Tris-HCl pH8.0) was added. The mixture was then loaded into Microcon Ultracel YM-10 filtration device and centrifuged at $14,000 \times \mathrm{g}$ for $15 \mathrm{~min}$. The concentrates were then diluted with $200 \mathrm{ul}$

UA buffer and centrifuged at $14,000 \times \mathrm{g}$ for $15 \mathrm{~min}$. After centrifugation, the concentrates were alkylated in 100ul IAA (50 mM IAA in UA) for 30 min in dark. After centrifuged for 10 min, the concentrates were 
washed twice with UA buffer and twice with $100 \mathrm{mM} \mathrm{NH4HCO3.} \mathrm{Subsequently,} \mathrm{trypsin} \mathrm{solution} \mathrm{(8 \mu g}$ Trypsin in $40 \mu \mathrm{L} \mathrm{NH} 4 \mathrm{HCO} 3$ buffer) was added to the filter and proteins were incubated at $37^{\circ} \mathrm{C}$ overnight. Tryptic peptides were collected by centrifugation followed by an additional wash with an elution solution (70\% ACN, $0.1 \%$ formic acid). Finally, the peptide mixture was desalted with C18-SD Extraction Disk Cartridge and the peptide concentration was assayed by measuring absorbance at $280 \mathrm{~nm}$.

\section{Tandem Mass Tagging Labeling}

Three control samples and 3 SAHA treatment samples (100 $\mu \mathrm{g}$, each) were labelled by TMT 6-plex reagents (Thermo Fisher Scientific) with TMT126, TMT127, TMT128, TMT129, TMT130, TMT131, respectively. Each sample was combined with its respective 6-plex TMT reagent and incubated for $1 \mathrm{~h}$ at room temperature. Then, hydroxylamine was added to the sample and incubated for 15 min to quench the reaction. Equal amounts of each TMT-labelled sample were combined in new microcentrifuge tubes and lyophilized in a SpeedVac concentrator.

\section{Phosphorylated and Acetylated peptides Enrichment}

Phosphopeptide enrichment were performed as described by Larsen et al. Briefly, Lyophilized peptides were re-suspended in DHB buffer ( $3 \% \mathrm{w} / \mathrm{v}$ DHB, $80 \% \mathrm{v} / \mathrm{v}$ ACN, $0.1 \% \mathrm{v} / \mathrm{v}$ trifluoroacetic acid (TFA)). Then, titanium dioxide beads (GL Sciences, Japan) were added and the mixture was agitated for $40 \mathrm{~min}$. TiO2 beads were recovered by centrifugation and washed three times with washing buffer I ( $30 \% \mathrm{ACN}$ and $3 \%$ TFA) and three times with washing buffer II ( $80 \%$ ACN and $0.3 \%$ TFA). Lastly, the phosphopeptides were eluted with elution buffer ( $5 \% \mathrm{NH} 4 \mathrm{OH} / 50 \% \mathrm{ACN})$, followed by lyophilization and $\mathrm{MS}$ analysis.

Prior to acetylated peptides enrichment, anti-lysine acetylation (Kac) antibody beads (PTM Biolabs, Inc, Hangzhou) was washed twice with ice-cold PBS. To enrich Kac peptides, 5 mg tryptic peptides of Kac was dissolved in NETN buffer (100 mM NaCl, 1 mM EDTA, $50 \mathrm{mM}$ Tris-HCl, 0.5\% NP-40, pH 8.0) and incubated with pre-washed antibody beads (Catalog No. PTM-104, PTM Biolabs, Inc, Hangzhou) in a ratio of $15 \mathrm{~mL}$ beads $/ \mathrm{mg}$ protein at $4^{\circ} \mathrm{C}$ overnight with gentle shaking. The beads were washed four times with NETN buffer and twice with ddH2O. The bound peptides were eluted from the beads with $0.1 \%$ TFA. The eluted peptides were collected and vacuum-dried followed by LC-MS/MS analysis.

\section{LC - ESI - MS/MS Analysis by Q-Extractive MS}

Peptides were dissolved in solvent A (0.1\% FA), loaded onto a Thermo scientific EASY column (C18 column, $5 \mu \mathrm{m}, 100 \mu \mathrm{m} \times 2 \mathrm{~cm}$, Thermo Scientific). Peptide separation was performed using a reversedphase analytical column (C18 column, $75 \mu \mathrm{m} \times 250 \mathrm{~mm}, 3 \mu \mathrm{m}$, Thermo Scientific). The gradient was comprised of an increase from $0-55 \%$ solvent $B(0.1 \%$ FA in $98 \%$ ACN) for $220 \mathrm{~min}, 55-100 \%$ for 8 min and then holding at $100 \%$ for the last $12 \mathrm{~min}$, at a constant flow rate of $250 \mathrm{nl} / \mathrm{min}$ on an EASY-nLC 1000 UPLC system. The eluted peptides were analyzed by Q Exactive ${ }^{\mathrm{TM}}$ hybrid quadrupole-Orbitrap mass spectrometer (Thermo Scientific). A data-dependent procedure was one MS scan (m/z range of 3501800) followed by $10 \mathrm{MS} / \mathrm{MS}$ scans for the top 20 precursor ions. Dynamic exclusion was enabled with exclusion duration of $30 \mathrm{~s}$. Automatic gain control (AGC) was set as $3 \mathrm{e} 6$ to prevent overfilling of the ion 
trap. The peptides were detected in the Orbitrap at a resolution of 70,000. Peptides were selected for MS/MS using NCE setting as 29 and ion fragments were detected at a resolution of 17,500.

\section{MS data analysis}

MS/MS spectra were searched using Mascot 2.2 (Matrix Science) embedded in Proteome Discoverer 1.4 against the UniProt human FASTA (released at 5/5/2018). For protein identification, the following options were used: peptide mass tolerance, $20 \mathrm{ppm}$; MS/MS tolerance, $0.1 \mathrm{Da}$; enzyme, trypsin; missed cleavage, 2; Fixed modifications, Carbamidomethyl (C); Variable modifications, TMT 6 plex (N-term) ,TMT6plex (K), Oxidation (M) $\square$ phosphorylation $(\mathrm{S} / \mathrm{T} / \mathrm{Y})$ and false discovery rate $(\mathrm{FDR}) \leq 0.01$. Proteome Discoverer 1.4 was used to extract the peak intensity of each expected TMT reporter ion from fragmentation spectrum. Only spectra in which all quant channels are present were used for quantification. The score threshold for peptide identification was set at a 1\% FDR and with phosphorylated and acetylated sites probability cutoff of 0.75 . Student's $T$ test was used to evaluate the statistical significance, and FDR was calculated. The criteria for significant abundance changes were the abundance ratios $\geq 1.2$ and the P-value $\leq 0.05$.

\section{Bioinformatic analysis}

Gene Ontology (GO) term association and enrichment analysis were performed using the Database for Annotation, Visualization and Integrated Discovery (DAVID). Encyclopedia of Genes and Genomes (KEGG) database was used to identify enriched pathways by Functional Annotation Tool of DAVID against the background of Homo sapiens. InterPro database was researched using Functional Annotation Tool of DAVID against the background of Homo sapiens. Manually curated CORUM protein complex database for human was used for protein complex analysis. To construct a protein-protein interaction network, the STRING database system was used. Functional protein interaction networks were visualized using Cytoscape. When performing the bioinformatics analysis, corrected $p$-value, 0.05 was considered significant. And all the detailed description of bioinformatic analysis was listed in Supplementary Information.

\section{Results}

\section{SAHA treatment changes proteome profile in NPC cells}

It has been reported that SAHA altered the global proteome in several kinds of cancers to deploy antitumor activity $[23,24]$. In this study, 6391 proteins were quantified in 5-8F cell line upon SAHA treatment. Among the 6391 quantified proteins, 454 were up-regulated and 217 were down-regulated with 1.2 change folds.

To illuminate the functions of the differentially expressed proteins (DEPs), performed comprehensive bioinformatics analyses were performed via Gene Ontology (GO) analysis, protein domain analysis, and Kyoto Encyclopedia of Genes and Genomes (KEGG) pathway analysis (Fig. 1A-1E). For the molecular function (MF) category, the DEPs were highly enriched in binding, catalytic activity and structural molecule activity (Fig. 1A). The analysis of cellular compartment (CC) displayed that proteins, which are 
involved in cell part, organelle and protein containing complex, were enriched toward SAHA treatment (Fig. 1B). Biological process (BP) analysis revealed that DEPs were mainly associated with cellular process, metabolic process biological process and cellular component organization (Fig. 1C).

Protein specific structural domain is one of its major functional characteristics. Consequently, we investigated the enriched domains of quantified proteins upon SAHA induction (Fig. 1D). We found that those domains related to ubiquitin-conjugating enzyme active site, nucleotide binding, histone $\mathrm{H} 1 / \mathrm{H} 5$ domain and RNA recognition motif domain were highly enriched.

To further explore the relevant pathways regulated by SAHA treatment, KEGG pathway analysis was performed. As shown in Fig. 1E, spliceosome, endocytosis and ubiquitin-mediated proteolysis pathways were the three most robustly enriched ones in response to SAHA stimulation, indicating the function of SAHA in regulating the signallings.

Finally, we established the protein protein interaction network via STING database and visualized by Cytoscape software (Fig. 1F). The global network of PPI and the highly enriched MAPKinase signaling pathway were presented. MAPK signaling, which regulates gene expression, cellular growth and survival, plays a vital role in tumorigenesis. It has been demonstrated that dysregulation of it may lead to abnormal cell proliferation and resistance to apoptosis $[25,26]$. This result indicated that MAPK signaling pathway might be a potential target of SAHA in NPC cells.

\section{SAHA treatment changes acetylome profile in NPC cells}

Given that SAHA is a pan HDAC inhibitor, we suspected that it will alter lysine acetylation in NPC cells. To this end, we performed quantitative acetylomics toward SAHA treatment in 5-8F cells by combination of TMT labeling, antibody enrichment of acetylation and LC-MS/MS analysis. Altogether, 441 lysine acetylation sites locating in 298 proteins were identified, of which 333 sites locating in 228 proteins were quantified. Within these quantified acetylation sites, 32 sites distributed on 26 proteins were upgraded and 47 sites distributed on 45 proteins were downward upon SAHA treatment (fold change $>1.2$ or $<-1.2$ ). And top ten acetylated sites and corresponding proteins upon SAHA stimulation were concluded ( Table 1). 
Table 1

Top ten acetylated sites and corresponding proteins with the highest change folds in acetylome upon SAHA treatment in 5-8F cells.

\begin{tabular}{|llll|}
\hline Symbol & Position & Fold change & Acetylated Probility \\
\hline PHIP & 1399 & 26.26 & AYTPSK(1)R \\
\hline PLXNB2 & 941 & 25.59 & VTK(1)FGAQLQCVTGPQATR \\
\hline SLC25A5 & 105 & 24.57 & QIFLGGVDK(1)R \\
\hline ALDOA & 147 & 23.78 & DGADFAK(1)WR \\
\hline CREBBP & 1595 & 23.2 & TNK(1)NKSSISR \\
\hline CREBBP & 1597 & 23.2 & TNKNK(1)SSISR \\
\hline NOLC1 & 251 & 23.15 & KQVVAK(1)APVK \\
\hline CTTN & 272 & 22.99 & TGFGGK(1)FGVQSER \\
\hline MACROH2A1 & 142 & 22.7 & SPSQK(0.964)KPVSK \\
\hline CTTN & 235 & 22.4 & GFGGK(1)FGVQTDR \\
\hline ACTN4 & 497 & -21.86 & CQK(1)ICDQWDALGSLTHSR \\
\hline AHNAK & 4239 & -21.9 & VDIDVPDVNIEGPDAK(1)LK \\
\hline KMT2A & 1130 & -21.98 & SSIAGSEDAEPLAPPIK(1)PIKPVTR \\
\hline AHNAK & 1305 & -22.07 & VDVEVPDVSLEGPEGK(1)LK \\
\hline SF3A1 & 486 & -22.32 & RTDIFGVEETAIGK(1)K \\
\hline MRPL47 & 144 & -22.36 & VVDSMDALDK(1)VVQER \\
\hline CLTA & 242 & -22.37 & SVLISLK(1)QAPLVH \\
\hline HSPD1 & 473 & -22.95 & TLK(1)IPAMTIAK \\
\hline SET & 7 & -23.9 & SAPAAK(1)VSKK \\
\hline EAF1 & 150 & -25.11 & APTK(1)PPVGPK \\
\hline
\end{tabular}

To understand features of these acetylated proteins, integrated bioinformatics analyses by combination with motif analysis, GO category analysis, KEGG pathway and protein-protein interaction analysis were conducted. As shown in Fig. 2A, we studied the amino acids which located around acetylated sites via motif analysis approach. In total, 6 motifs were robustly enriched: KacL, EKac, Kac**R, KacF, KacL, and $\mathrm{KacW}$ (Kac refers to acetylated lysine, * refers to random amino acid site). These 6 motifs differed in abundance and KacL, EKac, Kac** R comprised approximately $66 \%$ in all quantified peptides (Fig. 2B). SAHA altered histone acetylation, which has a great impact on chromatin remodeling and epigenetics 
[27]. Accordingly, distribution of acetylated proteins across chromosome was analyzed. This result demonstrated that these proteins were mainly located at 11 and 14 chromatins (Fig. 2C).

When GO database was applied to analyze these acetylated proteins, we found that these proteins mainly participated in regulation of RNA splicing process, ribonucleotide triphosphate metabolic process and purine ribonucleotide triphosphate metabolic process with biological process (BP) category analysis (Fig. 3A, top panel). In terms of molecular function (MF) category, chromatin DNA binding, nuclear receptor transcription coactivator activity and nucleosome binding are the top three items (Fig. 3A, middle panel). And as shown in cellular component (CC) analysis, these acetylated DEPs mainly involved in spliceosome complex, catalytic step 2 spliceosome and mitochondrial protein complex (Fig. 3A, bottom panel). These results revealed that SAHA may regulate RNA splicing process, metabolic-related process and chromatin DNA binding activity to play anti-tumor activities in NPC cells.

Next, KEGG pathway was conducted to have a look into the associated cellular signaling toward SAHA treatment. Totally, based on the criteria: $p<0.05$ and FDR $<0.05$, the DEPs took part in 30 prevalent cellular signaling (Fig. 3B). Among them, MYC, EGFR, mRNA splicing, G2M checkpoint, oxidation phosphorylation and TCA cycle were mainly enriched pathways, which were closely associated with proliferation, cellular metabolism and cell cycle processes. It has been well characterized that these notable processes are hallmarks of cancer [28]. These results hinted that SAHA may modulate these tumor-associated signaling pathways via protein acetylation to display treatment function in NPC cells.

In Fig. 3C, we clustered the differentially expressed acetylated proteins via two-way hierarchical clustering, which clearly displayed the patterns of the acetylated and global proteins in response to SAHA treatment. Taking advantage of STING database PPI network was studied. Representative MYC and EGFR signaling pathway were showed in Fig. 3D. In the MYC signaling pathway, there were 14 sites exhibiting decrease at acetylation level upon SAHA treatment, while 4 sites exhibited increase. For EGFR signaling pathway, there were 15 proteins quantified to be acetylated, 6 of which were upregulated and 9 were downregulated (Fig. 3D).

\section{Crosstalk between global proteome and acetylome in NPC cells}

According to the whole proteome and acetylome data from 5-8F cells upon SAHA treatment, the crosstalk between these two modifications were analyzed. In total, 126 proteins were quantified by proteome and acetylome, including 14 DEPs (Fig. 4A and 4B). Subsequently, these 14 DEPs, showing the highest significance and change fold values, were selected for two-way hierarchical clustering analysis (Fig. 4C). Followed by, the protein-protein interaction analysis was studied to unveil the functional relationship between these two profiles and representative interacted proteins were displayed in Fig. 4D. Together, these analyses indicated a complex relationship between proteome and acetylome, which in turn determine the functions of SAHA in NPC cells. 


\section{SAHA treatment changes phosphorylome profile in NPC cells}

Phosphorylation is one of the most important PTMs and is believed to take part in the process of cancer progression [12]. In previous study, we demonstrated that SAHA regulated the phosphorylation of p53 and Rb1 in NPC cells, hence protein phosphorylome towards SAHA stimulation was investigated here.

Classification and enrichment analysis based on GO revealed that the DEPs in phosphorylome were mainly associated with RNA related processes in biological process (BP) category, such as RNA localization process, nucleobase-containing compound transport process, establishment of RNA localization process and RNA/mRNA transport process. The cellular component (CC) category revealed that DEPs were primarily in chromosome, including chromosome, centromeric region, condensed chromosome and heterochromatin. For the molecular functional (MF) category, mRNA binding, histone binding, modification dependent protein binding and RNA polymerase binding were highly enriched (Fig. 5A).

To further study the related cellular signaling of phosphorylated DEPs, we conducted signaling analysis via KEGG database. In total, 30 significant cellular pathways were detected under the criteria $p<0.05$ and FDR $<0.05$ (Fig. 5B). It showed that cell cycle and mRNA splicing-related signaling pathways were robustly affected, which included G2M checkpoint pathway, M phase, mitotic spindle pathway and premRNA/mRNA splicing pathway. Intriguingly, SUMOylation-related signaling was enriched in SAHAinduced phosphorylome profile, which consisted with the previous study that SAHA regulated protein Sumoylation to implement biological functions.

Then distribution of phosphorylated proteins across chromosome was also studied. The result presented that enhanced phosphorylated proteins were mainly gathered in No.16 and No.2 chromatins, while decreased phosphorylated proteins mostly located at No.11 and No.19 chromatins (Fig. 5C).

We next established the protein-protein interaction network about phosphorylated proteins. The overview network of PPI and the representative mRNA export process were presented in Fig. 5D. The result uncovered that SAHA boosted some proteins' phosphorylation level (RANBP2, POM121, NUP214, SRSF10 and RNPS1), while reduced phosphorylation state of other proteins (SRSF5, NUP210, NUP188, NUP98, NUP107, NUP133 and SMG6) (Fig. 5D). Taken together, our results indicated that SAHA may manipulate the phosphorylation of some critical molecules to regulate pivotal signal pathways, leading to the therapeutic roles in NPC cells.

\section{Crosstalk between the global proteome and phosphorylome in NPC cells}

Based on the results of proteome and phosphorylome toward SAHA treatment in 5-8F cells, we found that 833 proteins in the global proteome were also phosphorylated, while 140 DEPs were detected to undergo the two modifications at the same time (Fig. 6A and 6B). We next assessed the correlation within the two 
modifications. The Pearson's correlation coefficient ratio $R^{2}$ was -0.16 , which suggested that global proteome and phosphorylome had weak negative relation in this situation (Fig. 6C). Following cellular signaling pathway analysis via KEGG database, we observed that several pathways were enriched in both two modifications, including cell cycle related and mRNA process related pathways (Fig. 6D). To evaluate the relevance between proteome and phosphorylome, we analyzed the protein-protein interaction network. The whole view of PPI between the global proteome and phosphorylome was showed in Fig. 6E. The complicated data hinted that there was closed crosstalk between global proteome and phosphorylome. Taken together, these results gave a hint that those important cellular processes and proteins underwent both proteome and phosphorylation might affect the functions of SAHA in NPC cells.

\section{Crosstalk between phosphorylome and acetylome in NPC cells}

It was reported that each protein modification can crosstalk with one or more other modifications to affect cellular functions [29]. As the most prominent two PTMs, phosphorylation and acetylation were demonstrated that linked closely with each other. To this point, we compared the acetylation and phosphorylation data from 5-8F cells treated with SAHA and 46 proteins were identified, which were both acetylated and phosphorylated (Fig. 7A). From the scatterplot, the connection ratio is 0.0319 , which meant the two protein modifications were not directly linked in the overall $\left(R^{2}=0.0319\right.$, Fig. 7B).

To better understand the relationship about these two protein modifications, we conducted the interaction network of proteins. The overall diagram of protein interaction was presented in Fig. 7C. Then through clustering these proteins, we found out that both acetylated and phosphorylated proteins taking part in regulating those prevalent biological processes, such as gene expression, epigenetics, chromatin organization, chromatin assembly and disassembly processes (Fig. 7D). For molecular functional (MF) analysis, mRNA binding, histone binding, chromatin DNA binding and histone acetyltransferase activity were mostly clustered items. Subsequently, those proteins were detected that mainly located in chromosome, nuclear periphery, heterochromatin and acetyltransferase complex (Fig. 7D). The compressive analyses suggested that these cellular processes were mediated both by acetylation and phosphorylation, thus affecting SAHA's activity.

\section{Discussion}

NPC is a malignant tumor of head and neck with poor prognosis. Although great progress has been made in treatment, especially in radiotherapy, the five years overall survival rate is still low due to the recurrence and metastasis [30]. It is urgent to develop predictive prevention and personalized medicine for NPC patients. Early prediction, prevention, targeted and personalized treatment, which are all key concepts in PPPM, are the main tools to improve the outcome of NPC patients [31]. SAHA has been demonstrated that implements anti-tumor effect in various cancers, including NPC [32]. However, the underlying mechanisms of its anti-tumor activity are still not well clear. In the current work, we utilized a TMT-based 
quantitative proteomic method, which integrated the data from proteome, acetylome and phosphorylome, to study the anti-tumor effect of SAHA in NPC cells.

Since SAHA belongs to deacetylase inhibitor family, we performed acetylome in 5-8F cells. The quantitative acetylation data revealed that 333 acetylated sites in 228 proteins were detected following SAHA treatment. From these acetylated sites, 32 sites of 26 proteins were upregulated and 47 sites of 45 proteins were downregulated. These apparently changed proteins took part in a variety of biological functions, of which MYC and EGFR pathways ranked the top two enriched pathways (Fig. 3B). Both have been well characterized in the progression of various cancers.

MYC is one of the most highly amplified oncogenes, which is deregulated among diverse human cancers, including myeloma, head and neck tumors, lymphoma and breast cancer [33]. Hence, alterations of MYC are commonly found on the path to cancer. It demonstrated that K-Ras conferred SAHA resistance by upregulating HDAC6 and MYC expression in colon cancer cells [34]. Wajana L. etc. reported that MYC controlled the sensitivity of gastric cancer upon HDAC inhibitors via directly regulating MCL1 and elF4E gene transcription and combination of MYC inhibitor and HDAC inhibitor could be a better therapeutic option for some gastric cancer patients [35]. As the expression and activity of MYC are dynamically affected by PTMs, therapeutics targeting at these protein modifications provide an innovative, efficient way to reduce MYC functions in cancers. There were researches reported that kinases induced phosphorylation of MYC and enzymes that affected MYC ubiquitin-dependent proteolysis could serve as the potential targets [36]. Moreover, MYC inhibitors and HDAC inhibitors, such as SAHA, may have cross effects to fight against cancers. Thus, in the combination of these two kinds of inhibitors might be a promising therapeutic choice for cancer patients [37]. Consistent with these researches, our present results uncovered that SAHA treatment significantly altered the acetylation level of MYC pathway in 5-8F cells. This might be one of the prominent principles of SAHA to play roles in NPC cells, while further validation needs to be scheduled. Moreover, our study provided useful resources to further investigate the cross-effects between HDAC inhibitors and MYC inhibitors.

The epidermal growth factor receptor (EGFR) exerts critical functions in epithelial cell physiology, which belongs to the ErbB family of receptor tyrosine kinases (RTKs) [38]. Its deregulation, such as mutation and/or overexpression, was reported in different types of human cancers, including head and neck cancer. Thus, currently EGFR has been a promising target of multiple cancer therapies. He L. etc. revealed that TSA, a HDAC inhibitor, decreased the EGFR-Arf1 signaling to inhibit cell migration and invasion in SCCHN [39]. Another group demonstrated that SAHA, in combination with Gefitinib, displayed synergistic anti-tumor activities in SCCHN cell lines via disturbing EGFR receptor expression [26]. In addition to head and neck cancer, it was reported that HDAC inhibitor alone was able to block both EGFR and HDAC in colorectal cancer, and may provide more benefits to the development of CRC therapy [40]. These reports supported our finding that SAHA treatment significantly affected EGFR signaling pathway via PTMs in NPC cells, which gave us a much deeper insight into the rationale of SAHA's effects in cancers. However, further investigations need to be done to validate the data and to explore the mechanism of SAHA deregulating EGFR pathway in NPC cells. 
Protein functions are mediated by a number of PTMs, which subsequently manipulate key cellular processes [41]. Phosphorylation and acetylation are the two most prevalent PTMs in the eukaryotic proteome [42]. In the present study, we performed the global proteome, acetylation and phosphorylation in 5-8F cells following SAHA stimulation. Our results showed that besides the well-established effects on protein acetylation, SAHA also regulated the global proteome and phosphorylation. Considering the growing evidence indicated that the link between acetylation and phosphorylation, it is reasonable to analyze the crosstalk among global proteome, acetylation and phosphorylation toward SAHA stimulation in NPC cells. According to our data, SAHA treatment directly altered protein acetylation and phosphorylation, the overlap between the two modifications were mainly related to the gene expression, chromatin organization, chromatin assembly, mRNA binding and histone binding process. Some proteins were acetylated and phosphorylated at the same time in these networks, which indicated that these proteins might play critical effects in regulating SAHA activities in cancer cells, therefore, their biological functions should be further investigated. In agreement with our study, Peter C. etc. found that phosphorylation and acetylation were synergistic coupling in response to EGF stimulation, H3 phosphorylation can affect the efficiency of acetylation reactions [43].

Cellular metabolism is one of the prominent hallmarks of cancer [28]. In this study, our data showed that protein acetylation was robustly enriched in the TCA cycle and glycolysis process following SAHA stimulation via bioinformatics analyses. It has been reported that protein acetylation regulated cellular metabolism in liver tissue, including the TCA cycle and glycolysis [44]. Our findings showed that the acetylation of some metabolic enzymes and metabolic-related processes were altered toward SAHA treatment in NPC cells, which established a link between acetylation and enzymes that catalyze metabolic processes.

\section{Conclusions And Expert Recommendations}

In this study, taking the advantages of TMT labeling, TiO2 enrichment, acetylated antibody enrichment and high-resolution LC-MS/MS, we presented a large-scale quantitative analysis of the global proteome, acetylome and phosphorylome in NPC cells in response to SAHA treatment. Our work provided a precious database that will contribute to the development of predictive and personalized practice in NPC. Furthermore, the correlation among the global proteome, phosphorylome and acetylome expanded our understanding of SAHA-induced cancer treatment, which is expected to be helpful for the PPPM practice in NPC patients.

We recommend that multiomics analysis, including proteome, acetylome and phosphorylome, is a potent tool to uncover the underlying mechanism of SAHA. Sequentially it drives the swift progress of PPPM treatment in NPC patients. Meanwhile we also suggest that further validations of those targeted genes and signaling pathways, like the EGFR signaling pathway, MYC signaling pathway, TCA cycle and glycolysis process, need to be employed.

\section{Abbreviations}


PPPM

Predictive preventive personalized medicine

SAHA

Suberoylanilide hydroxamic acid

HDACs

Histone deacetylases

NPC

Nasopharyngeal carcinoma

FDA

Food and Drug Administration

CTCL

Cutaneous T cell lymphomas

EBV

Epstein-Barr virus

PTMs

Protein posttranslational modifications

LC-MS/MS

Liquid chromatography-tandem mass spectrometry

IMAC

Immobilized metal affinity chromatography

TiO2

Titanium dioxide

DEPs

Differentially expressed proteins

\section{Declarations}

\section{COMPETING INTERESTS}

The authors declare that they have no competing interests.

\section{FUNDING}

The present study was supported by the National Natural Science Foundation of China (No. 81974074, 81570537 and 81902858) and Major State Basic Research Development Program of China (No. 2014CBA02000-4).

\section{DATA AVALIABILITY STATEMENT}

The mass spectrometry proteomics data have been deposited to the ProteomeXchange Consortium (http://proteomecentral.proteomexchange.org) via the iProX partner repository with the dataset identifier PXD021029. 
CODE AVILIABILITY

All software applications used are included in this article.

\section{AUTHORS' CONTRIBUTIONS}

Huichao Huang, Ying Fu, Maoyu Li and Yongheng Chen designed the study and conceived the experiments; Huichao Huang and Maoyu Li performed the experiments; Ye Zhang, Fang Peng, and Ying Fu provided technical and material support; Huichao Huang, Miaolong Lu, and Zhuchu Chen analyzed and interpreted the data; Huichao Huang and Maoyu Li wrote the manuscript. All authors read and approved the final manuscript.

\section{ACKNOWLEDGEMENTS}

We thank Dr. Jun Zhao for the constructive comments on this manuscript.

\section{CONSENT FOR PUBLICATION}

Not applicable

\section{References}

1. Shahbazian MD, Grunstein M. Functions of site-specific histone acetylation and deacetylation. Annu Rev Biochem. 2007;76:75-100.

2. Wagner JM, et al. Histone deacetylase (HDAC) inhibitors in recent clinical trials for cancer therapy. Clin Epigenetics. 2010;1(3-4):117-36.

3. Marks PA, Breslow R. Dimethyl sulfoxide to vorinostat: development of this histone deacetylase inhibitor as an anticancer drug. Nat Biotechnol. 2007;25(1):84-90.

4. Duvic $\mathrm{M}$, et al. Phase 2 trial of oral vorinostat (suberoylanilide hydroxamic acid, SAHA) for refractory cutaneous T-cell lymphoma (CTCL). Blood. 2007;109(1):31-9.

5. Wu Q, et al. Suberoylanilide hydroxamic acid treatment reveals crosstalks among proteome, ubiquitylome and acetylome in non-small cell lung cancer A549 cell line. Sci Rep. 2015;5:9520.

6. Konstantinopoulos PA, et al. Suberoylanilide hydroxamic acid (SAHA) enhances olaparib activity by targeting homologous recombination DNA repair in ovarian cancer. Gynecol Oncol. 2014;133(3):599-606.

7. Komatsu N, et al. SAHA, a HDAC inhibitor, has profound anti-growth activity against non-small cell lung cancer cells. Oncol Rep. 2006;15(1):187-91.

8. Munster PN, et al. The histone deacetylase inhibitor suberoylanilide hydroxamic acid induces differentiation of human breast cancer cells. Cancer Res. 2001;61(23):8492-7.

9. Xu G, et al. SAHA regulates histone acetylation, Butyrylation, and protein expression in neuroblastoma. J Proteome Res. 2014;13(10):4211-9. 
10. Hoffman MD, Sniatynski MJ, Kast J. Current approaches for global post-translational modification discovery and mass spectrometric analysis. Anal Chim Acta. 2008;627(1):50-61.

11. Blixt $O$, et al. Printed covalent glycan array for ligand profiling of diverse glycan binding proteins. Proc Natl Acad Sci U S A. 2004;101(49):17033-8.

12. Krueger KE, Srivastava S. Posttranslational protein modifications: current implications for cancer detection, prevention, and therapeutics. Mol Cell Proteomics. 2006;5(10):1799-810.

13. Sheehan KM, et al. Use of reverse phase protein microarrays and reference standard development for molecular network analysis of metastatic ovarian carcinoma. Mol Cell Proteomics. 2005;4(4):34655.

14. Oliveira AP, Sauer $U$. The importance of post-translational modifications in regulating Saccharomyces cerevisiae metabolism. FEMS Yeast Res. 2012;12(2):104-17.

15. Tripodi F, et al. Post-translational modifications on yeast carbon metabolism: Regulatory mechanisms beyond transcriptional control. Biochim Biophys Acta. 2015;1850(4):620-7.

16. Vlastaridis P, et al., The Pivotal Role of Protein Phosphorylation in the Control of Yeast Central Metabolism. G3 (Bethesda), 2017. 7(4): p. 1239-1249.

17. Drazic A, et al. The world of protein acetylation. Biochim Biophys Acta. 2016;1864(10):1372-401.

18. Habibian J, Ferguson BS. The Crosstalk between Acetylation and Phosphorylation: Emerging New Roles for HDAC Inhibitors in the Heart. Int J Mol Sci, 2018. 20(1).

19. Park S, et al. Degradation or aggregation: the ramifications of post-translational modifications on tau. BMB Rep. 2018;51(6):265-73.

20. Kishi $\mathrm{H}$, et al. Osmotic shock induces $\mathrm{G} 1$ arrest through p53 phosphorylation at Ser33 by activated p38MAPK without phosphorylation at Ser15 and Ser20. J Biol Chem. 2001;276(42):39115-22.

21. Golubnitschaja 0 , et al. Position paper of the EPMA and EFLM: a global vision of the consolidated promotion of an integrative medical approach to advance health care. Epma j. 2013;4(1):12.

22. Seredin P, et al. Pathology-specific molecular profiles of saliva in patients with multiple dental cariespotential application for predictive, preventive and personalised medical services. Epma j. 2018;9(2):195-203.

23. Wu Q, et al. SAHA treatment reveals the link between histone lysine acetylation and proteome in nonsmall cell lung cancer A549 Cells. J Proteome Res. 2013;12(9):4064-73.

24. Garmpis N, et al. Histone Deacetylases as New Therapeutic Targets in Triple-negative Breast Cancer: Progress and Promises. Cancer Genomics Proteomics. 2017;14(5):299-313.

25. Citro $S$, et al. Synergistic antitumour activity of HDAC inhibitor SAHA and EGFR inhibitor gefitinib in head and neck cancer: a key role for $\Delta$ Np63a. Br J Cancer. 2019;120(6):658-67.

26. Bruzzese $F$, et al. HDAC inhibitor vorinostat enhances the antitumor effect of gefitinib in squamous cell carcinoma of head and neck by modulating ErbB receptor expression and reverting EMT. J Cell Physiol. 2011;226(9):2378-90. 
27. Kim S, Kaang BK. Epigenetic regulation and chromatin remodeling in learning and memory. Exp Mol Med. 2017;49(1):e281.

28. Hanahan D, Weinberg RA. Hallmarks of cancer: the next generation. Cell. 2011;144(5):646-74.

29. Hunter T. The age of crosstalk: phosphorylation, ubiquitination, and beyond. Mol Cell. 2007;28(5):730-8.

30. Cao SM, Simons MJ, Qian CN. The prevalence and prevention of nasopharyngeal carcinoma in China. Chin J Cancer. 2011;30(2):114-9.

31. Cheng T, Zhan X. Pattern recognition for predictive, preventive, and personalized medicine in cancer. Epma j. 2017;8(1):51-60.

32. Huang $\mathrm{H}$, et al. Dissection of Anti-tumor Activity of Histone Deacetylase Inhibitor SAHA in Nasopharyngeal Carcinoma Cells via Quantitative Phosphoproteomics. Front Cell Dev Biol. 2020;8:577784.

33. Dang CV. MYC on the path to cancer. Cell. 2012;149(1):22-35.

34. Wang Q, et al. Oncogenic K-ras confers SAHA resistance by up-regulating HDAC6 and c-myc expression. Oncotarget. 2016;7(9):10064-72.

35. Labisso WL, et al. MYC directs transcription of MCL1 and elF4E genes to control sensitivity of gastric cancer cells toward HDAC inhibitors. Cell Cycle. 2012;11(8):1593-602.

36. Sears R, et al. Multiple Ras-dependent phosphorylation pathways regulate Myc protein stability. Genes Dev. 2000;14(19):2501-14.

37. Allen-Petersen BL, Sears RC. Mission Possible: Advances in MYC Therapeutic Targeting in Cancer. BioDrugs, 2019. 33(5): p. 539-553.

38. Schlessinger J. Receptor tyrosine kinases: legacy of the first two decades. Cold Spring Harb Perspect Biol, 2014. 6(3).

39. He L, et al. Histone deacetylase inhibitors suppress aggressiveness of head and neck squamous cell carcinoma via histone acetylation-independent blockade of the EGFR-Arf1 axis. J Exp Clin Cancer Res. 2019;38(1):84.

40. Chou CW, et al. HDAC inhibition decreases the expression of EGFR in colorectal cancer cells. PLoS One. 2011;6(3):e18087.

41. Swaney DL, et al. Global analysis of phosphorylation and ubiquitylation cross-talk in protein degradation. Nat Methods. 2013;10(7):676-82.

42. Kim SC, et al. Substrate and functional diversity of lysine acetylation revealed by a proteomics survey. Mol Cell. 2006;23(4):607-18.

43. Cheung $\mathrm{P}$, et al. Synergistic coupling of histone $\mathrm{H} 3$ phosphorylation and acetylation in response to epidermal growth factor stimulation. Mol Cell. 2000;5(6):905-15.

44. Zhao $S$, et al. Regulation of cellular metabolism by protein lysine acetylation. Science. 2010;327(5968):1000-4. 
Figures

A

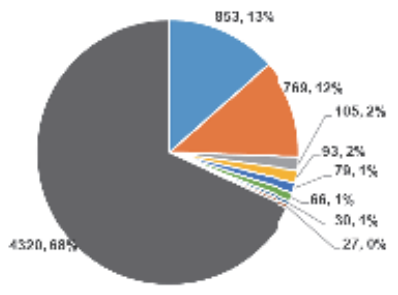

binding

ceatabnic assivity

- structural molecte actover

- molscular function reguester

- tansperoer activty

- mank:ríptise registex artivity

- translation reguder activity

- molecular transoucar activity

- ocher

c

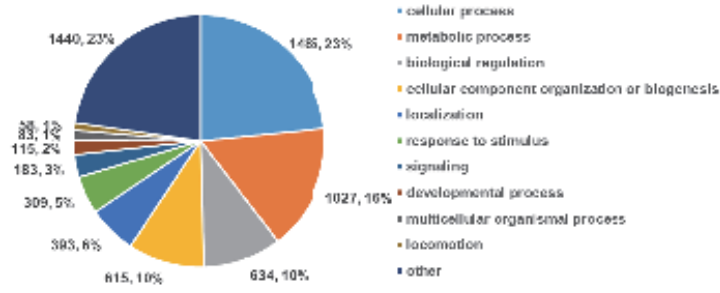

E

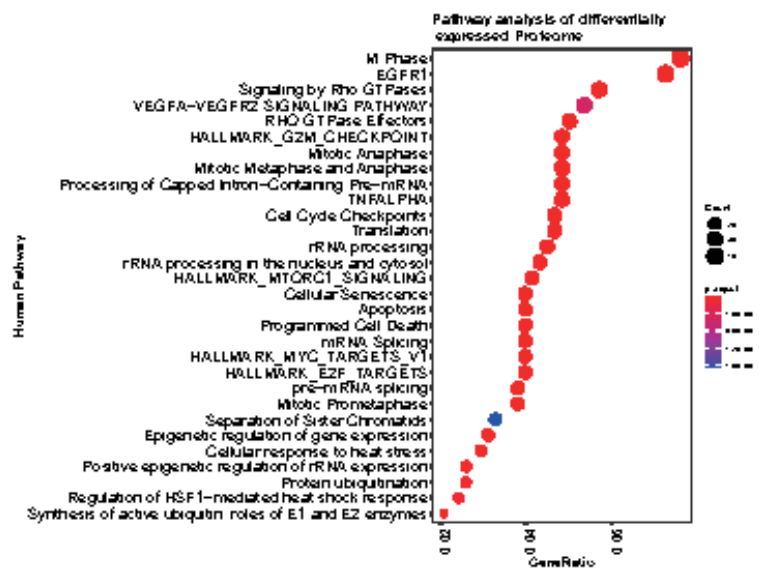

B

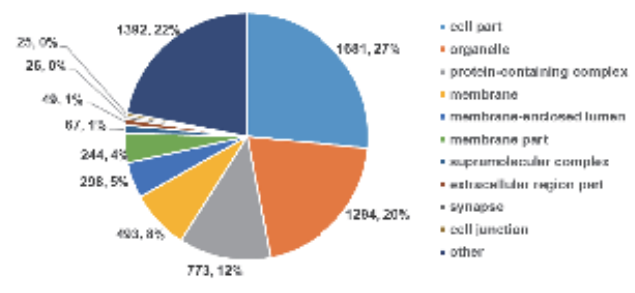

D

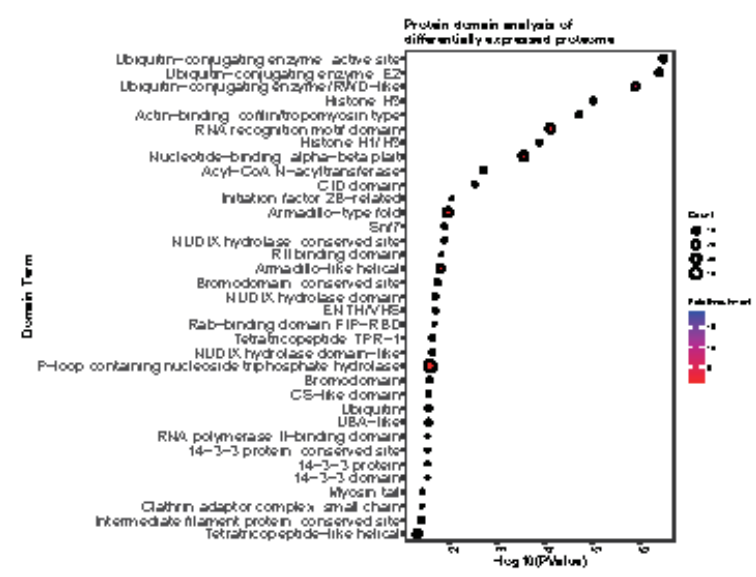

F

Figure 1. Functional enrichment cluster analysis of quantified proteome

\section{Figure 1}

Functional enrichment cluster analysis of quantified global proteome (A) Molecular function. (B) Cellular compartment. (C) Biological process. (D) Protein domain analysis. (E) KEGG pathway enrichment 
analysis of differentially expressed proteins. (F) Protein-protein interaction network of proteome. The global view and representative MAPK pathway were displayed.

A

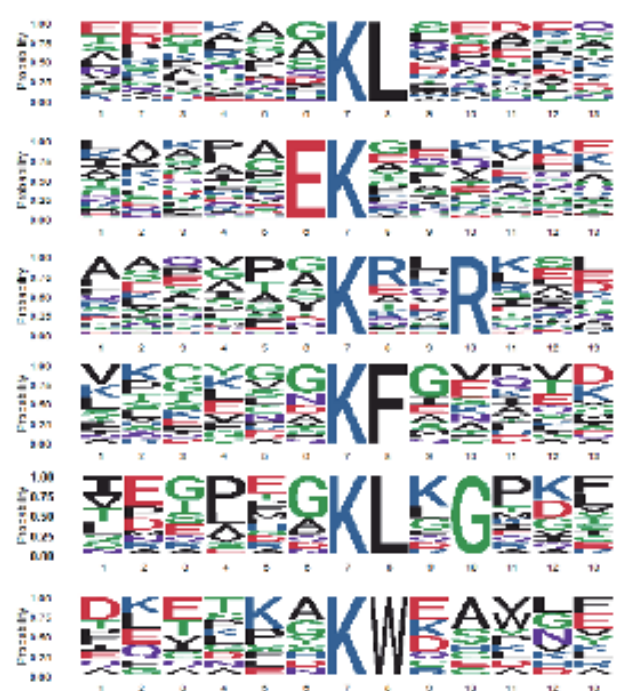

B

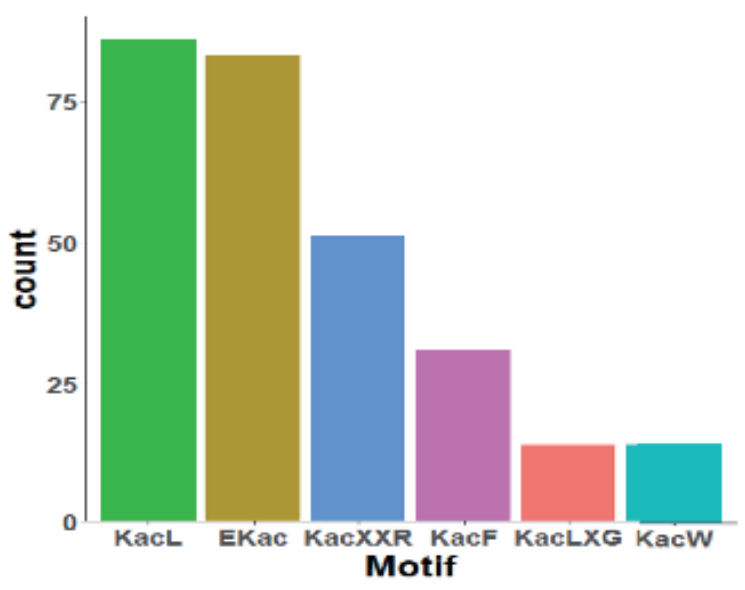

C

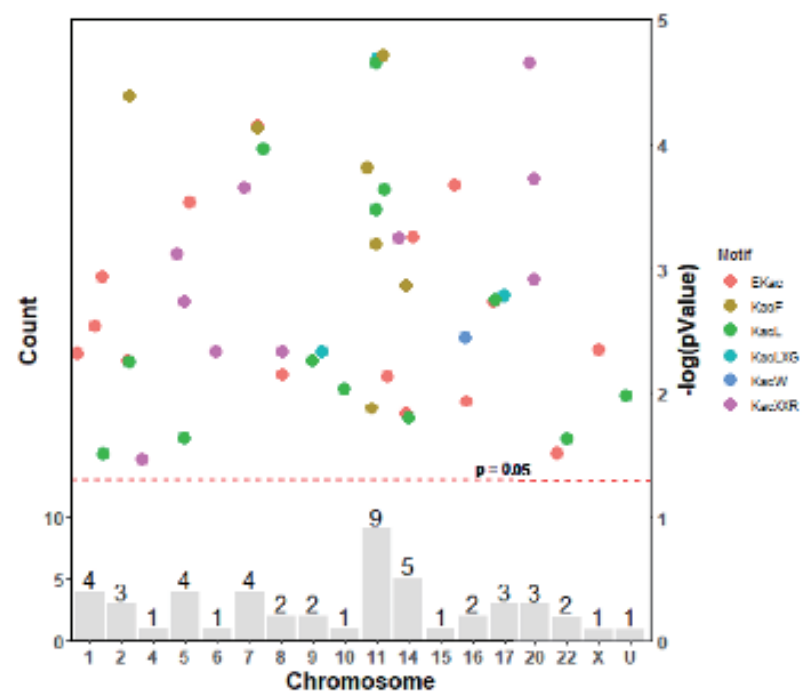

Figure 2. Motif analysis of the identified acetylation peptides in SAHA-treated cells

Figure 2

Motif analysis of the identified acetylation peptides in SAHA-treated cells (A) Sequence logo of acetylated motifs. (B) Number of identified peptides containing acetylated lysine in each motif. (C) Distribution of acetylated proteins across chromosomes. The red dashed line corresponds to $\mathrm{P}=0.05$, and the peptide 
sites (dots $\llbracket$ above this threshold are shown in different colors according to their motifs. The bars indicate the total number of acetylated sites in each chromosome. U: undetermined.

A

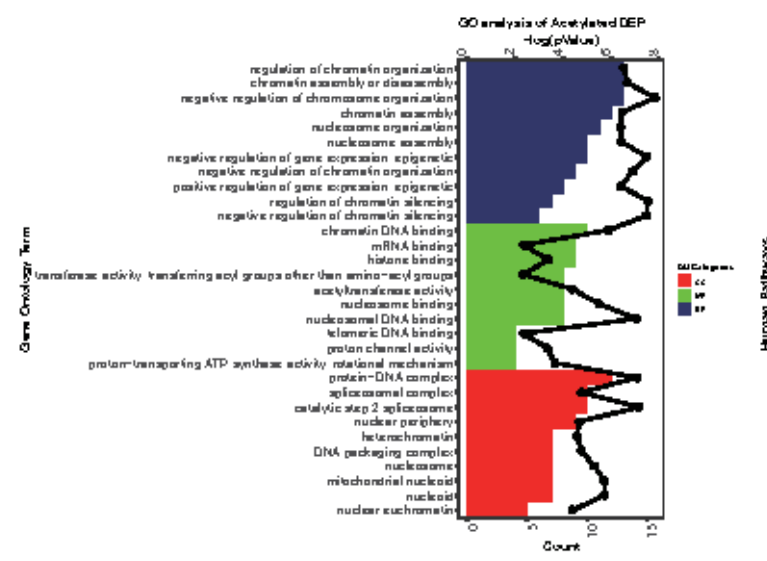

C

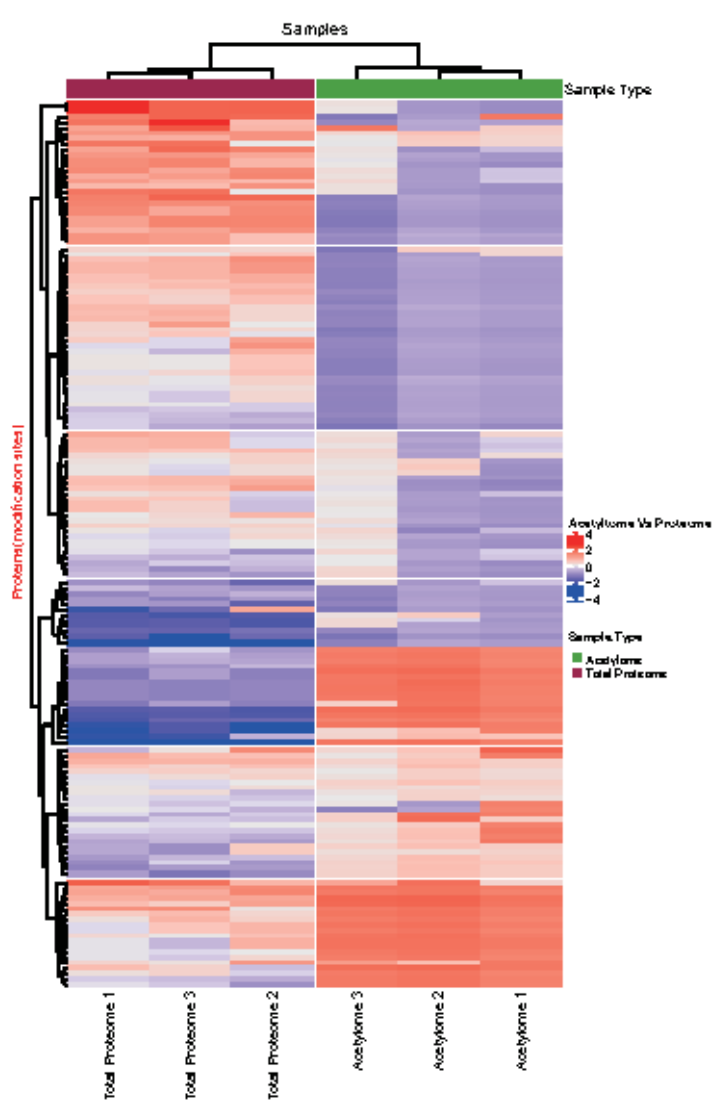

B

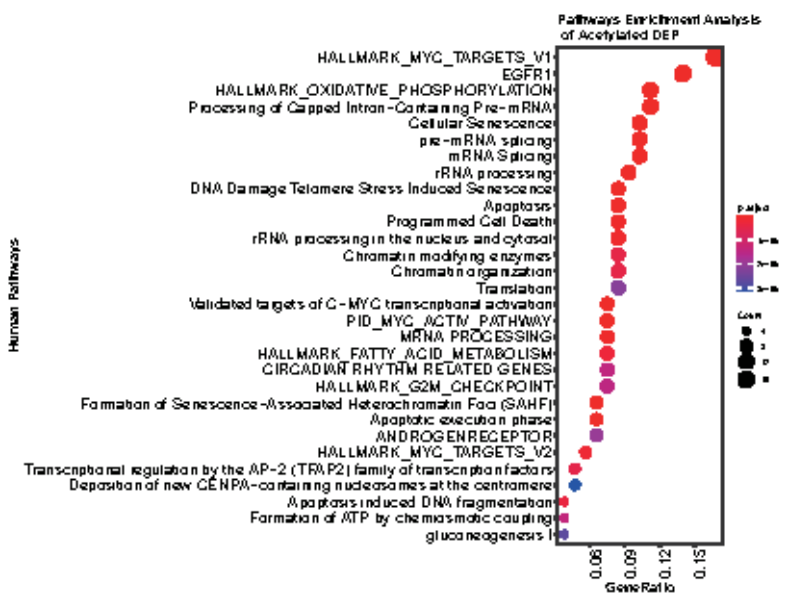

D
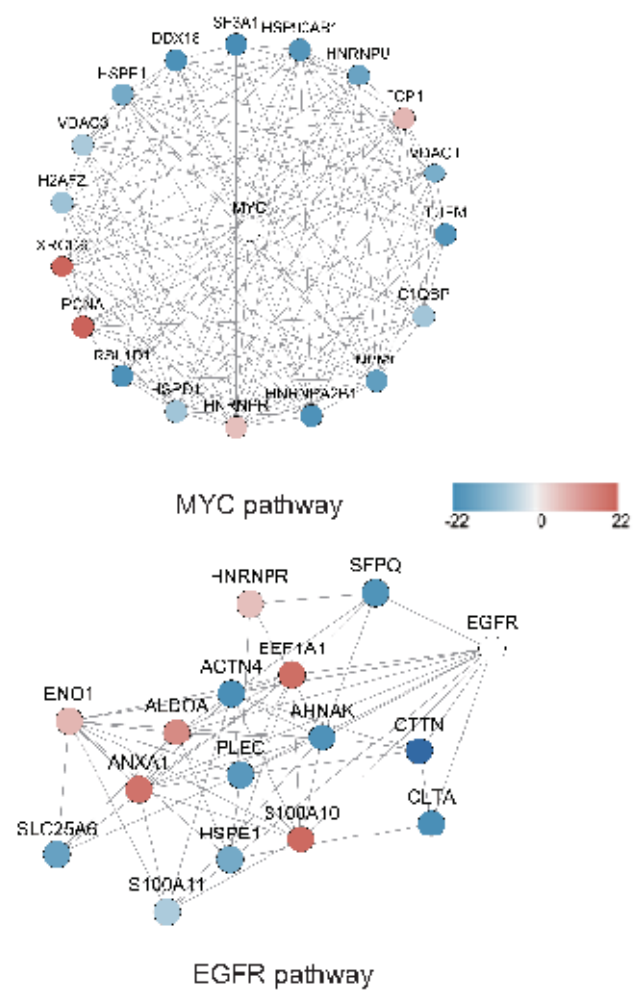

Figure 3. Functional enrichment analysis of quantified acetylated proteins

\section{Figure 3}

Functional enrichment analysis of quantified acetylated proteins (A) GO enrichment analysis. (B) KEGG pathway analysis. (C) Heatmap diagram of a two-way hierarchical clustering. The red and blue colors indicate expression level of proteins in terms of normalized ratio. Upper color labeling shows total 
proteome samples in red and acetylome in green. Distance of hierarchical clustering was measured using the Euclidean method. (D) Protein-protein interaction network of acetylome clustered in representative MYC and EGFR pathway.

A

Overlapping of Acetylome and Proteome

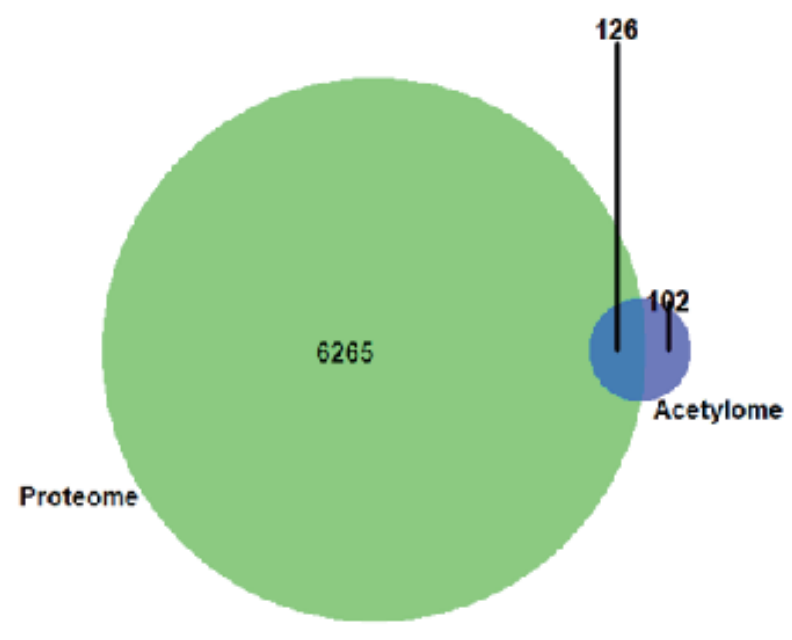

C

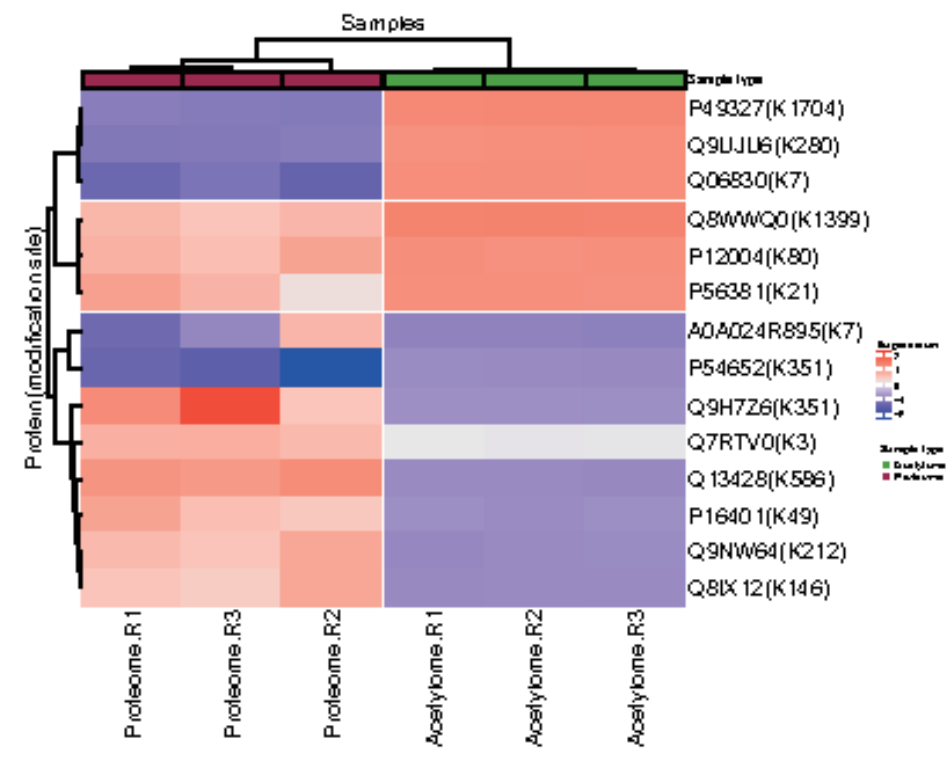

B

Overlapping of differentally expressed proteins between acetylome and proteome

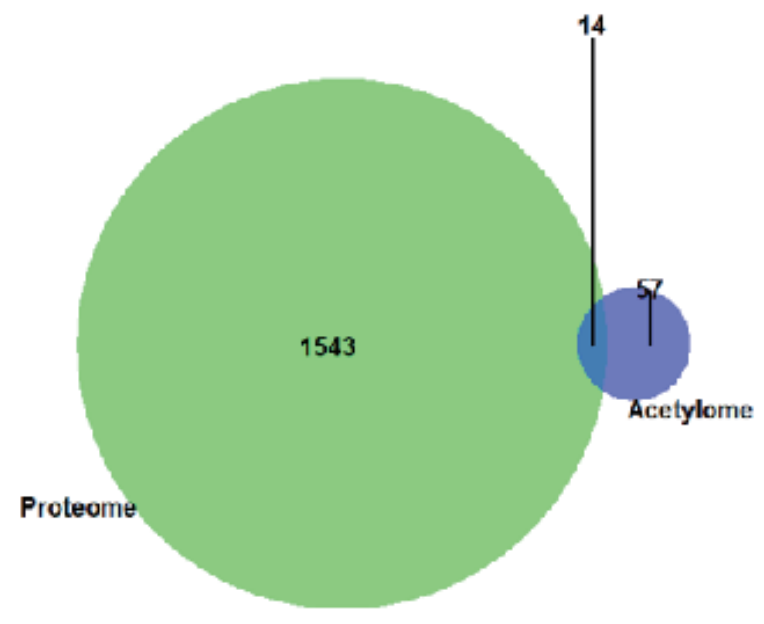

D

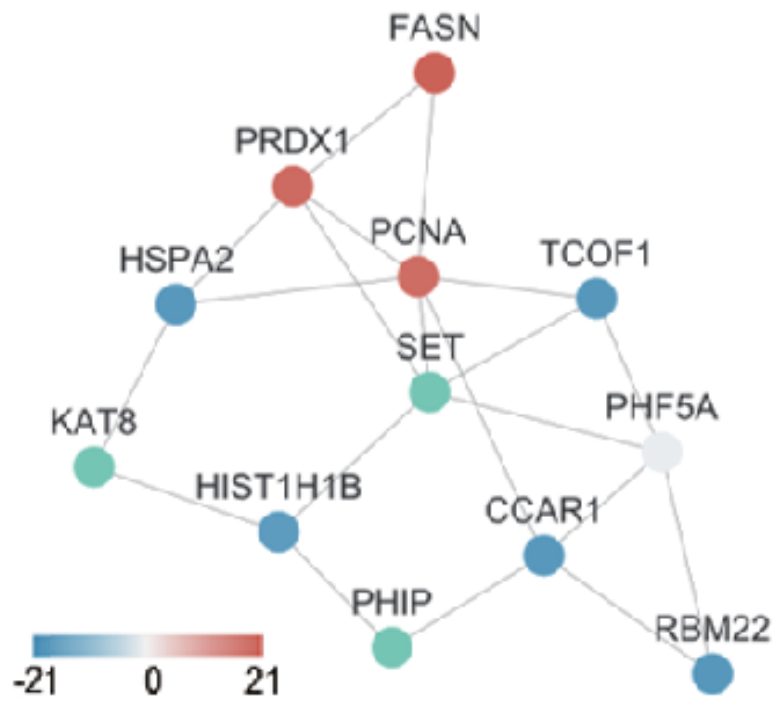

Figure 4. Functional analysis between proteome and acetylome

Figure 4

Functional analysis between proteome and acetylome (A) Overlap of total proteins between acetylome and proteome. (B) Overlap of differentially expressed proteins between acetylome and proteome. (C) 
Heatmap diagram of a two-way hierarchical clustering. Diagram consists of the 14 most differential expressed proteins in global proteome and acetylome. The red and blue colors indicate expression level of proteins in terms of normalized ratio. Upper color labeling shows total proteome samples in red and acetylome in green. Distance of hierarchical clustering was measured using the Euclidean method. (D) Protein-protein interaction network of differentially expressed proteins between acetylome and proteome.

A

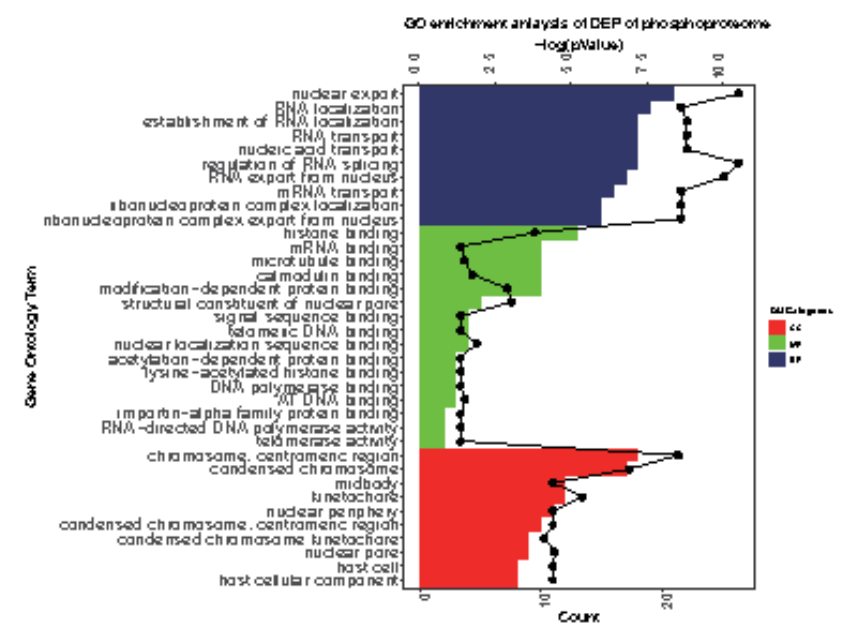

B

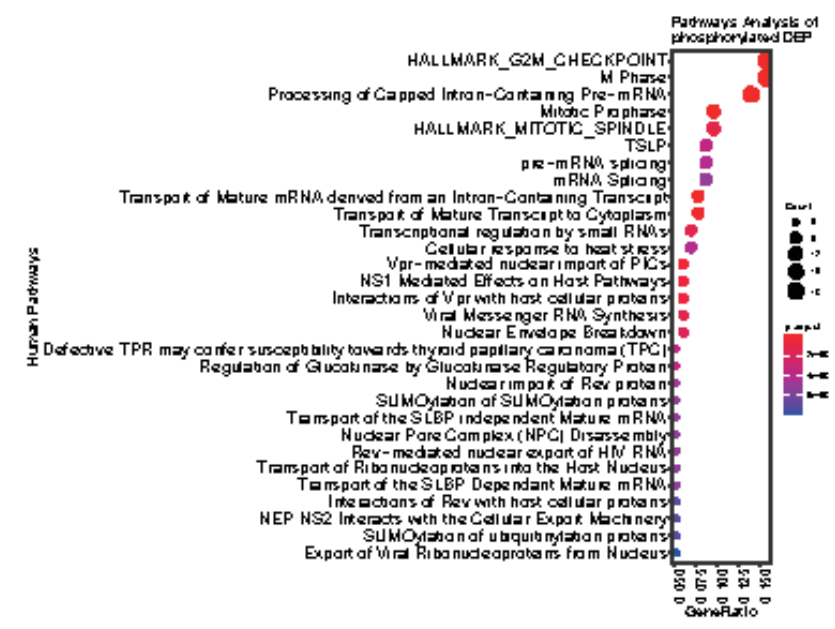

C
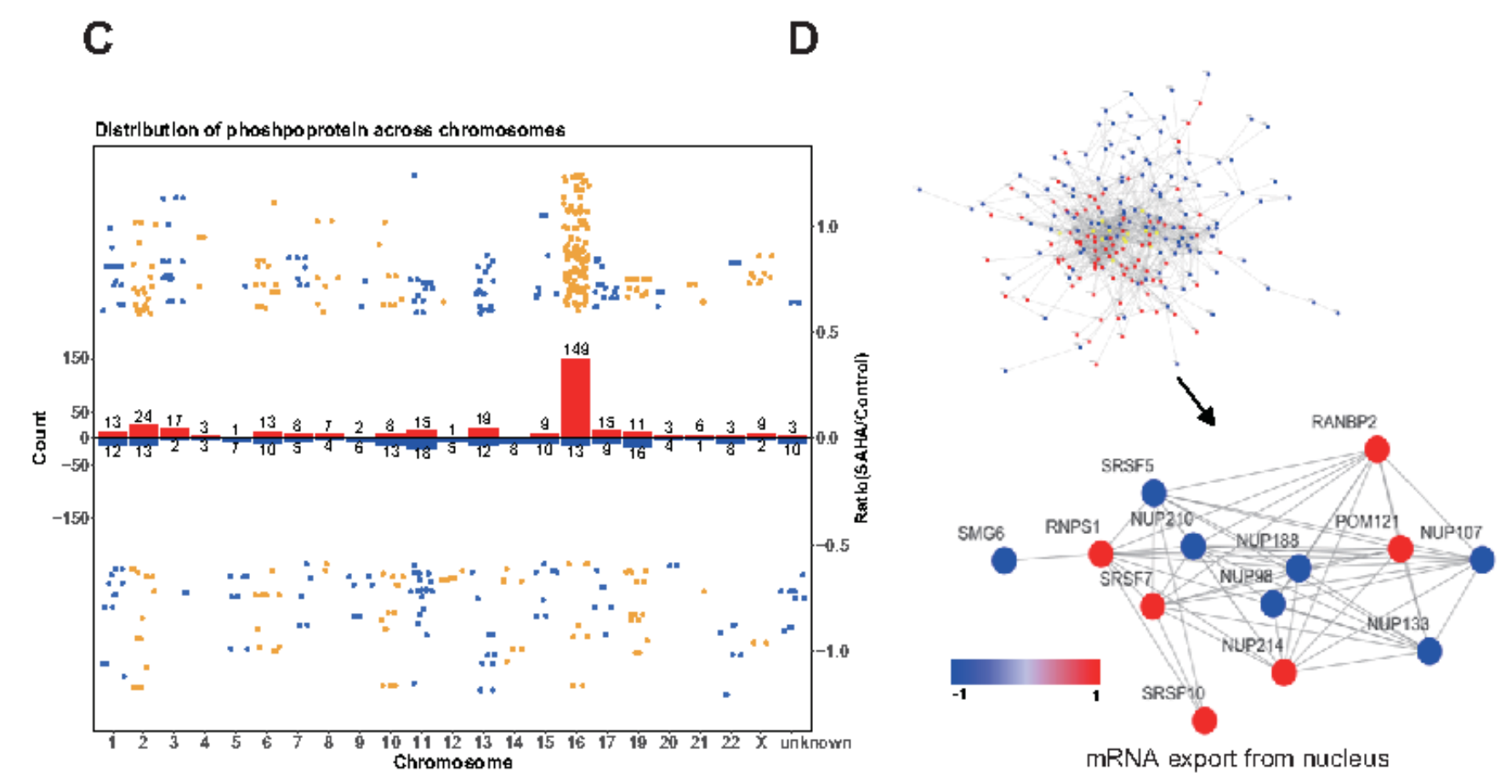

Figure 5. Functional enrichment analysis of quantified phosphorylated proteins

\section{Figure 5}


Functional enrichment cluster analysis of quantified phosphorylated proteins. (A) GO enrichment analysis. (B) KEGG pathway analysis. (C) Distribution of phosphorylated proteins across chromosomes. The number above each bar indicated the total number of phosphorylated proteins in each chromosome. Red bar refers to upregulated proteins; Blue bar refers to downregulated proteins. (D) Protein-protein interaction network of phosphorylome. Both global network and representative mRNA export process were displayed.

A Overlapping of total protein B between Phosphoproteome and Proteome
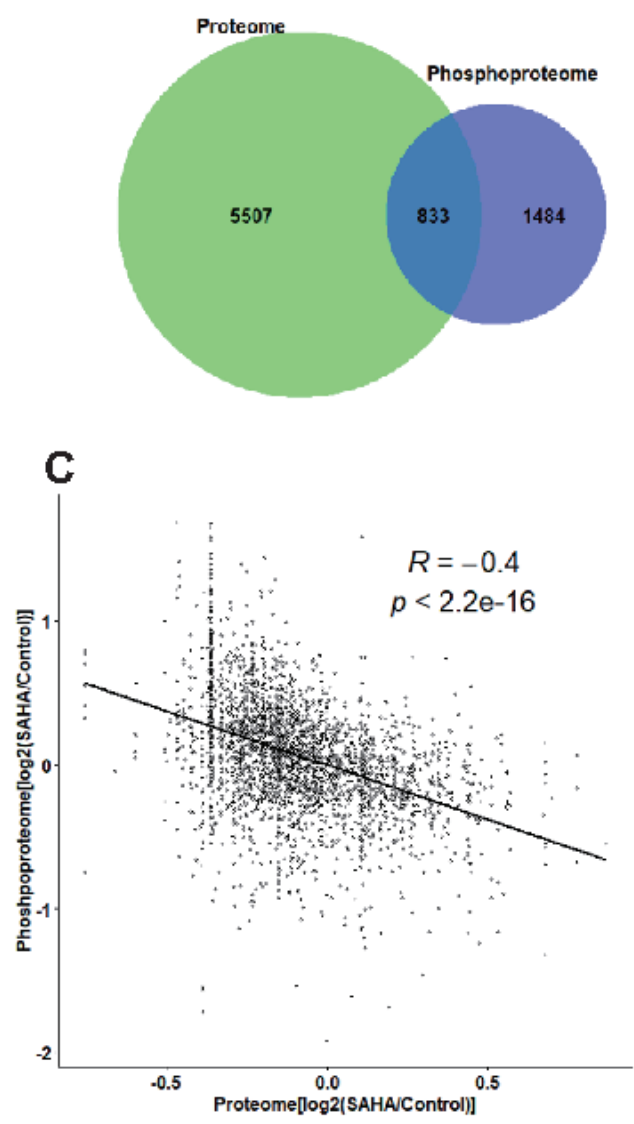

D

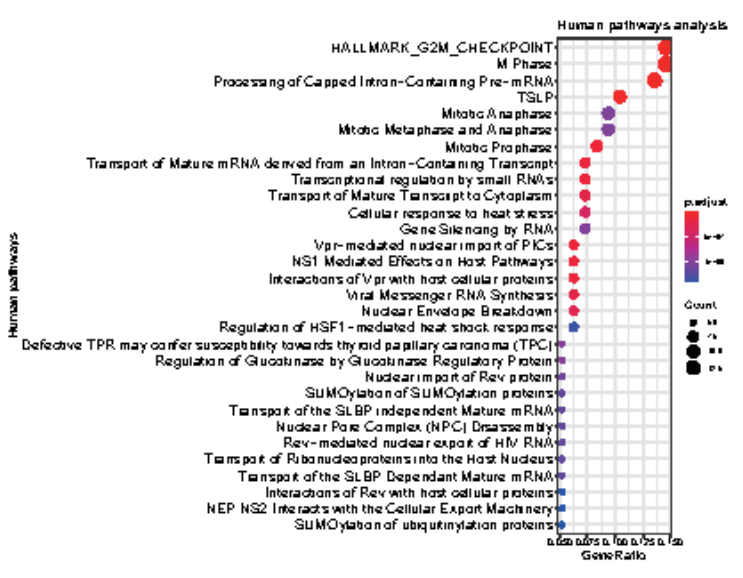

E

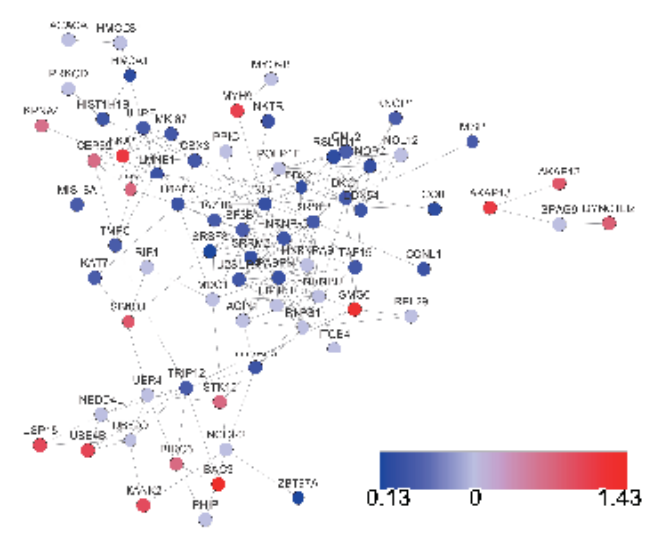

Figure 6. Crosstalk analysis between proteome and phosphorylome 
Crosstalk analysis between proteome and phosphorylome (A) Overlap of total proteins between phosphorylome and proteome. (B) Overlap of differentially expressed proteins between phosphorylome and proteome. (C) Correlation between phosphorylome and proteome. (D) KEGG pathway analysis. (E) Protein-protein interaction network of diferentially expressed proteins between phosphorylome and proteome.

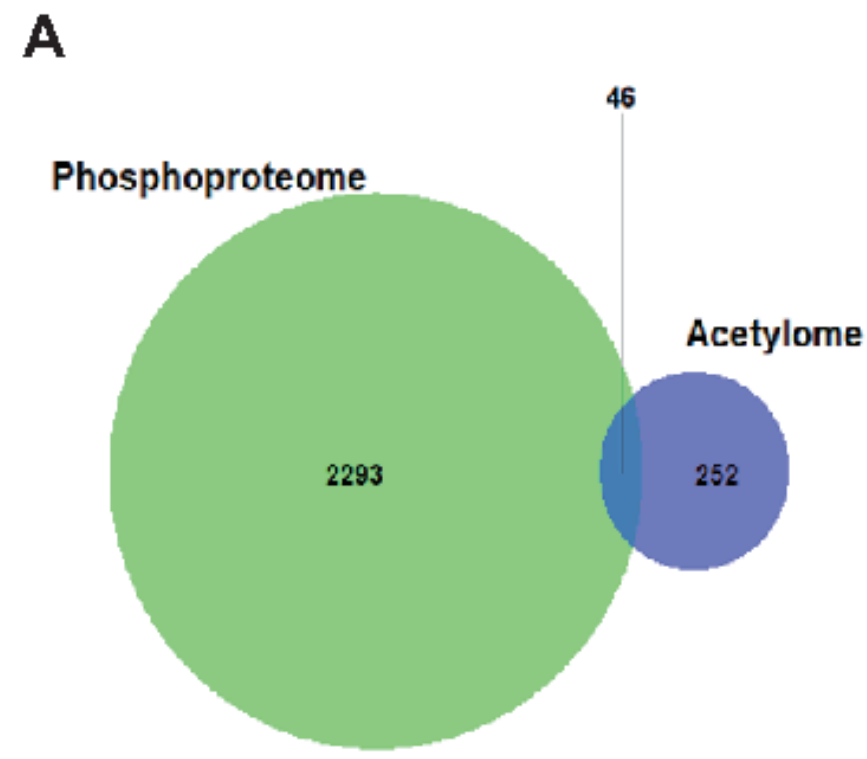

B

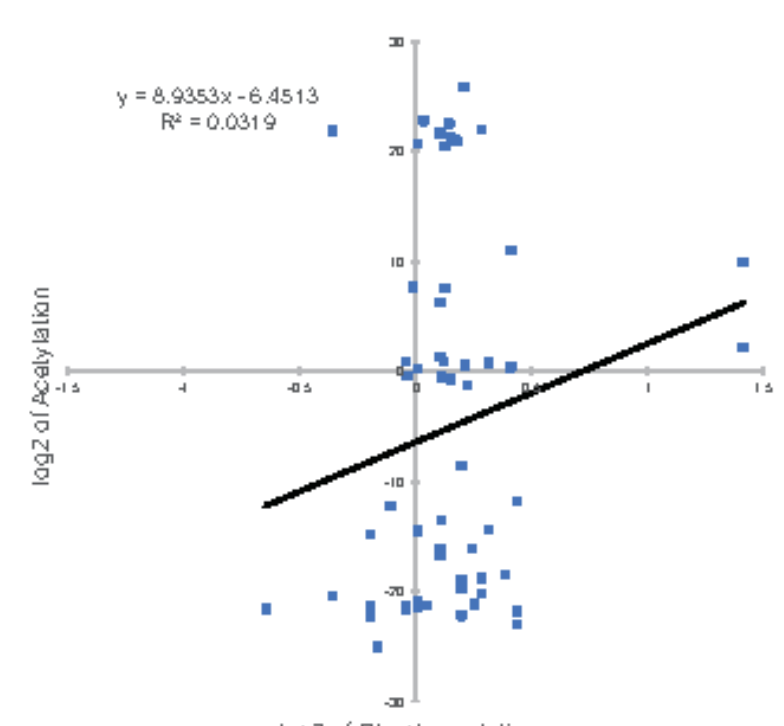

C

D
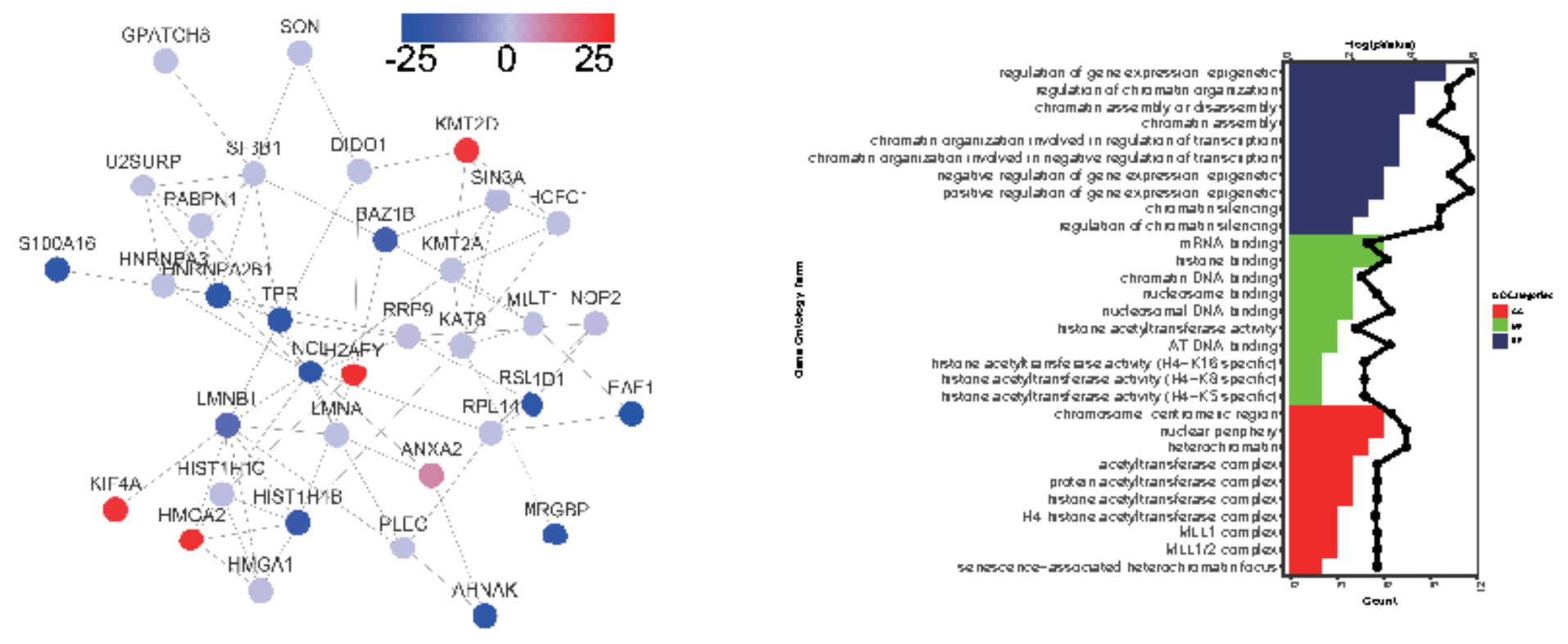

Figure 7. Crosstalk analysis between phosphorylome and acetylome

Figure 7 
Crosstalk analysis between phosphorylome and acetylome (A) Overlap of total proteins between acetylome and phosphorylome. (B) Correlation between acetylome and phosphorylome. (C) Proteinprotein interaction network between acetylome and phosphorylome. (D) GO enrichment analysis between acetylome and phosphorylome. 